\title{
Diversity of 16S rRNA Genes of New Ehrlichia Strains Isolated from Horses with Clinical Signs of Potomac Horse Fever
}

\author{
BOHAI WEN, ${ }^{1}$ YASUKO RIKIHISA, ${ }^{1 *}$ PAUL A. FUERST, ${ }^{2}$ AND WIWAT CHAICHANASIRIWITHAYA ${ }^{1}$ \\ Department of Veterinary Biosciences ${ }^{1}$ and Department of Molecular Genetics, ${ }^{2}$ \\ The Ohio State University, Columbus, Ohio 43210-1093
}

\begin{abstract}
Ehrlichia risticii is the causative agent of Potomac horse fever. Variations among the major antigens of different local $E$. risticii strains have been detected previously. To further assess genetic variability in this species or species complex, the sequences of the 16S rRNA genes of several isolates obtained from sick horses diagnosed as having Potomac horse fever were determined. The sequences of six isolates obtained from Ohio and three isolates obtained from Kentucky were amplified by PCR. Three groups of sequences were identified. The sequences of five of the Ohio isolates were identical to the sequence of the type strain of $E$. risticii, the Illinois strain. The sequence of one Ohio isolate, isolate 081 , was unique; this sequence differed in 10 nucleotides from the sequence of the type strain (level of similarity, 99.3\%). The sequences of the three Kentucky isolates were identical to each other, but differed by five bases from the sequence of the type strain (level of similarity, 99.6\%). The levels of sequence similarity of isolate 081 , the Kentucky isolates, and the type strain to the next most closely related Ehrlichia sp., Ehrlichia sennetsu, were 99.3, 99.2, and 99.2\%, respectively. On the basis of the distinct antigenic profiles and the levels of 16S rRNA sequence divergence, isolate 081 is as divergent from the type strain of $E$. risticii as $E$. sennetsu is. Therefore, we suggest that strain 081 and the Kentucky isolates may represent two new distinct Ehrlichia species.
\end{abstract}

Ehrlichia risticii is a small, gram-negative, obligately intracellular bacterium that causes Potomac horse fever, an acute systemic infectious disease of the family Equidae $(11,15)$. Since it was first recognized in 1979 along the Potomac River in Maryland and Virginia (7), Potomac horse fever has been shown to occur throughout North America $(10,11)$, in France (19), and possibly in India (15). E. risticii is antigenically and genetically most closely related to Ehrlichia sennetsu, the etiologic agent of human Sennetsu fever in Japan and Malaysia (1, $11,12,14,16)$. E. sennetsu can establish infections in horses but is not pathogenic, and preinoculation of E. sennetsu can protect horses from E. risticii challenge (14).

During clinical diagnostic work in our laboratory, many strains of $E$. risticii have been isolated from horses which have had clinical signs compatible with Potomac horse fever, and these isolates have been identified on the basis of morphological and serological criteria. The antigenic and morphological characteristics of six isolates obtained from infected horses residing in Ohio and three isolates obtained from horses residing in Kentucky have been studied previously (3). Antigenic diversity among these strains was detected by Western blot (immunoblot) analysis and indirect fluorescent-antibody (IFA) tests in which we used monoclonal antibodies to $E$. risticii Illinois or Maryland strains which were isolated from two different infected horses residing in Maryland in 1984. In particular, isolate 081 , which was obtained from an infected horse residing in Ohio, was antigenically unique. None of the 22 monoclonal antibodies tested reacted with isolate 081 as determined by the IFA assay (3). Furthermore, the three Kentucky isolates were similar to each other, but different from the Ohio isolates or the Illinois strain. The Ohio isolates were much more similar to Illinois and Virginia strains isolated from horses residing in Maryland in 1984 in their immunoblot pro-

\footnotetext{
* Corresponding author. Mailing address: Department of Veterinary Biosciences, College of Veterinary Medicine, The Ohio State University, 1925 Coffey Rd., Columbus, OH 43210-1093.
}

files and IFA reactivity with monoclonal antibodies than they were to either the Kentucky isolates or isolate 081 (3).

In order to study whether the antigenic diversity among strains that cause Potomac horse fever reflects strain differences within one Ehrlichia species or represents the results of divergence between closely related species, we sequenced and analyzed 16S rRNA genes amplified from the nine new isolates by PCR.

\section{MATERIALS AND METHODS}

Isolation of strains from horse blood. Blood samples (100 to $200 \mathrm{ml}$ ) from six clinically sick horses residing in Ohio and three horses residing in Kentucky that had been diagnosed as having Potomac horse fever (Table 1) were aseptically collected in sterile, heparinized $(5 \mathrm{U} / \mathrm{ml})$ syringes and centrifuged at $1,600 \times g$ for $10 \mathrm{~min}$. After the plasma was removed from each sample, the buffy coat was aspirated and layered on Histopaque 1077 (Sigma Chemical Co., St. Louis, Mo.), and then the preparation was centrifuged at $800 \times \mathrm{g}$ for $15 \mathrm{~min}$ at room temperature. The interface containing mononuclear cells was collected and washed in RPMI 1640 medium (GIBCO Laboratories, Grand Island, N.Y.) containing an antibiotic mixture $\left(10^{4} \mathrm{U}\right.$ of penicillin per $\mathrm{ml}, 1 \mathrm{mg}$ of streptomycin per $\mathrm{ml}, 25 \mathrm{U}$ of amphotericin per $\mathrm{ml}$; GIBCO) at a concentration of $1 \%$, and the resulting preparation was centrifuged at $1,000 \times g$ for $5 \mathrm{~min}$. The pellet containing leukocytes was overlaid on monolayers of P388D1 murine macrophage cells (American Type Culture Collection, Rockville, Md.) in RPMI 1640 medium supplemented with $10 \%$ heat-inactivated fetal bovine serum (GIBCO) and $2 \mathrm{mM}$ L-glutamine (GIBCO) without antibiotics in $25-\mathrm{cm}^{2}$ tissue culture flasks. The cells were incubated at $37^{\circ} \mathrm{C}$ in a humidified atmosphere containing $5 \% \mathrm{CO}_{2}$ and $95 \%$ air. After 1 or 2 days, floating lymphocytes were discarded. The samples of cultured cells were examined daily by making slides, using centrifugation (Cytospin; Shandon, Inc., Pittsburgh, Pa.) and Diff-Quik staining (Baxter Scientific Products, Obetz, Ohio), until the cultures were positive for intracellular infection. Infection was confirmed by transmission electron microscopy and IFA assay. The infected cultures were maintained by serial passages in P388D1 cells. All of the isolates used in this study were grown for less than 20 passages. The microorganisms were purified from the host cells by sonication, differential centrifugation, and chromatographic fractionation with Sephacryl S-1000 (Pharmacia, Uppsala, Sweden) as previously described (13)

Extraction of DNA. Approximately $10^{5}$ infected P388D1 cells resuspended in $1 \mathrm{ml}$ of TE buffer (40 mM Tris, $1 \mathrm{mM}$ EDTA; $\mathrm{pH} 8.0$ ) containing $1 \%$ sodium dodecyl sulfate and $20 \mu \mathrm{g}$ of proteinase $\mathrm{K}$ (Sigma) per ml were incubated at $50^{\circ} \mathrm{C}$ for $2 \mathrm{~h}$. The resulting lysed suspension was extracted twice with an equal volume of phenol-chloroform-isoamyl alcohol $(25: 24: 1)$ and once with an equal volume of chloroform-isoamyl alcohol (24:1). The DNA was subsequently precipitated from the resulting aqueous phase by adding 0.1 volume of $3 \mathrm{M}$ sodium acetate 
TABLE 1. Clinical data for horses from which organisms were isolated

\begin{tabular}{cllc}
\hline Horse & \multicolumn{1}{c}{ Clinical signs $^{a}$} & \multicolumn{1}{c}{ Location $^{b}$} & IFA titer $^{c}$ \\
\hline 022 & A, F, Di, Deh, L & Troy, Ohio & $1: 640$ \\
067 & A, De, Di, Deh & Alexandria, Ohio & $1: 640$ \\
081 & A, F, De, Di & Findley, Ohio & $1: 160$ \\
606 & A, F, Deh, L & Dover, Ohio & $1: 2,560$ \\
380 & A, F, Di & Hudson, Ohio & $1: 1,280$ \\
679 & A, F, Di, L & Athens, Ohio & $1: 5,120$ \\
Co & A, F, De, Deh, Di, L & Versailles, Ky. & $1: 320$ \\
Ov & A, F, Di, De, Deh & Versailles, Ky. & $1: 640$ \\
As & A, F & Versailles, Ky. & $1: 320$ \\
\hline
\end{tabular}

${ }^{a}$ Clinical signs at the time of blood collection. Abbreviations: A, anorexia; F, fever; De, depression; Deh, dehydration; Di, diarrhea; L, laminitis.

${ }^{b}$ Location of the horse during the month before the organism was isolated.

${ }^{c}$ IFA titer detected in the blood collected (the 1984 E. risticii Maryland strain was used as the reference antigen).

(pH 5.2) and 2.5 volumes of cold 95\% ethanol and was washed with ice-cold $70 \%$ ethanol after a brief centrifugation at $10,000 \times g$

Amplification of 16S rRNA. The 16S rRNA gene was amplified by using the flanking primers ER5' (5'-GATCCTGGCTACGAACG-3') and ER3' (5'-TA ACCCCAGTCACCCAC- $3^{\prime}$ ). Genomic DNA and 50 pmol of each primer were used in a $100-\mu \mathrm{l}$ reaction mixture which contained $10 \mu \mathrm{l}$ of $10 \times$ buffer, $5 \mu \mathrm{l}$ of 50 $\mathrm{mM} \mathrm{MgCl} 2,2 \mu \mathrm{l}$ of a $10 \mathrm{mM}$ deoxyribonucleotide triphosphate mixture, and 2.5 $\mathrm{U}$ of Taq DNA polymerase (GIBCO BRL, Gaithersburg, Md.). PCR was performed for 30 cycles, with each cycle consisting of $94^{\circ} \mathrm{C}$ for $1 \mathrm{~min}, 55^{\circ} \mathrm{C}$ for $2 \mathrm{~min}$, and $72^{\circ} \mathrm{C}$ for $3 \mathrm{~min}$. The expected DNA fragment $(\sim 1.5 \mathrm{~kb})$ was purified from a $1 \%$ low-melting-temperature agarose gel (GIBCO BRL) by using a PCR DNA purification system (Promega, Madison, Wis.). The purified double-stranded DNA was kept at $-20^{\circ} \mathrm{C}$ for sequencing.

DNA sequencing of $16 S$ rRNA. Sequencing was performed by the dideoxyribonucleotide method, using a double-stranded DNA cycle sequencing system as recommended by the manufacturer (GIBCO BRL). The sequencing primers were end-labelled with $\left[\gamma^{32} \mathrm{P}\right] \mathrm{ATP}$ by using T4 kinase (GIBCO BRL). A $5 \%$ Long Ranger gel (AT Biochem, Malvern, Pa.) was used for sequencing in order to determine the sequence of more than 400 bases for each primer. Both strands were sequenced with the following forward and reverse primers: ER5', ER3', ER 323-341 (5'-CTACGGGAGGCAGCAGTG-3'), ER757-775 (5'-TAGTCCACG CTGTAACG-3'), ER1043-1062 (5'-TAAGTCCCGCAACGAGC-3'), ER864846 (5'-GAGTTTTAGTCTTGCGACC-3'), ER493-476 (5'-GTATTACCGCG GCGCTG-3'), and ER247-228 (5'-CATTACCCCACCAACTAGC-3'). The primers were chosen by considering the 16S rRNA sequence of the $E$. risticii Illinois strain; the program OLIGO (National Biosciences, Inc., Hamel, Mich.) was used for analysis.

Computer analyses of DNA sequences. Sequences were aligned manually with the program ESEE (2). The corrected levels of nucleotide divergence for $16 \mathrm{~S}$ rRNA gene sequences were calculated by using DNADIST from PHYLIP, version $3.1(6)$. A phylogenetic tree based on the sequences was constructed by using the neighbor-joining method, implemented by NEIGHBOR in PHYLIP (6), and the distance matrix data. Only homologous sites within the 16S rRNA gene sequences that exhibited unambiguous alignment were included in the phylogenetic analysis. An alternative cladistic reconstruction of the phylogenetic relationships among taxa was also obtained by using parsimony (DNAPARS) from PHYLIP; this method produced results identical to the results obtained when the neighbor-joining method was used.

Additional taxa. Representative sequences obtained from other members of the tribe Ehrlichieae were included for comparison. The nucleotide sequences of the 16S rRNA genes of the following organisms were included in the analysis: $E$. sennetsu, E. risticii, Neorickettsia helminthoeca, an intracellular endosymbiont of Nasonia vitripennis (strain F), Anaplasma marginale, Ehrlichia equi, Ehrlichia canis, Cowdria ruminantium, and Rickettsia rickettsii.

Nucleotide sequence accession numbers. The accession numbers for the nucleotide sequences used for comparison in this study are as follows: $E$. sennetsu, M73225; E. risticii, M21290; Neorickettsia helminthoeca, U12457; Nasonia vitripennis intracellular endosymbiont strain F, M84688; Anaplasma marginale, M60313; E. equi, M73223; E. canis, M73221; C. ruminantium, X61659; and $R$. rickettsii, U11021.

\section{RESULTS}

Amplification of 16S rRNA genes. Using the flanking primers (ER5' and ER3'), we amplified only one band $(\sim 1.5 \mathrm{~kb})$ from each of the nine isolates studied. These results are consistent with the results obtained previously for isolates of sev-

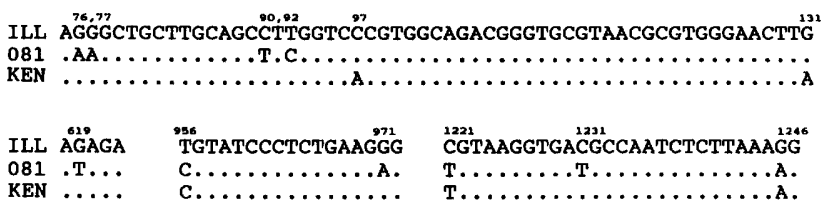

FIG. 1. Nucleotide substitutions in the 16S rRNA genes of Ohio isolate 081 , Kentucky isolates (obtained from horses Co, Ov, and As) (KEN), and ILL, the Illinois strain, and other Ohio isolates (obtained from horses 022, 067, 380, 606, and 679).

eral Ehrlichia species in our laboratory and in other laboratories $(1,4)$. The bands were isolated and purified from the agarose gel for sequencing.

Sequence analysis. The sequence flanked by our primers was 1,428 bases long for each amplimer. The sequences of five of the Ohio isolates were identical to the previously described sequence of the Illinois strain of E. risticii (20). However, the sequence of isolate 081 differed from the sequence of the Illinois strain at 10 nucleotide positions (level of similarity, $99.3 \%$ ). The sequences of the three Kentucky isolates did not differ from each other, but differed at five positions from the sequence of the Illinois strain (level of similarity, 99.6\%). The level of sequence divergence between isolate 081 and the Kentucky isolates was $0.6 \%$ (level of similarity, 99.4\%). The levels of sequence similarity of isolate 081 , the Kentucky isolates, and the Illinois strain to E. sennetsu were 99.3, 99.2, and 99.2\%, respectively. The variable regions of the 16S rRNA genes of the new isolates and the Illinois strain of E. risticii are shown Fig. 1.

The adjusted evolutionary distances used in our phylogenetic comparison of the 16S rRNA genes are shown in Table 2. An evolutionary tree which shows the relationships among the Kentucky strains, strain 081, and the closely related organisms E. risticii, E. sennetsu, and Neorickettsia helminthoeca, which was obtained by using the neighbor-joining method, is shown in Fig. 2. This tree is identical to the tree obtained when the parsimony method was used. Isolates 081 and As, a representative Kentucky isolate, are the most closely related organisms which make a cluster with $E$. risticii. The three organisms then make a cluster with $E$. sennetsu. These organisms and Neorickettsia helminthoeca form a distinct cluster within the $\alpha$ subclass of the Proteobacteria, which is well separated from other members of the tribe Ehrlichieae (Table 2).

\section{DISCUSSION}

Sequence comparison of 16S rRNA genes is recognized as one of the most powerful and precise methods for determining the phylogenetic relationships of bacteria $(8,9,21)$. In part, this is so because bacterial 16S rRNA genes evolve so slowly that there is little or no sequence divergence among strains belonging to one species. This implies that a small difference in gene sequences may indicate that organisms belong to different species (1). Although species recognition is usually supported by phenotypic and other genotypic criteria, the results of $16 \mathrm{~S}$ rRNA sequence analyses have been used as important criteria for identifying new isolates and naming new species $(1,4)$. This has been especially true for intracellular forms, such as Ehrlichia strains, which are difficult to grow and purify in sufficient quantity for biochemical and other types of genetic studies.

The sequences of the 16S rRNA genes of six Ohio isolates and three Kentucky isolates obtained from sick horses diagnosed as having Potomac horse fever were determined. On the basis of the results of a $16 \mathrm{~S}$ rRNA gene sequence analysis, the 
TABLE 2. Levels of genetic similarity and differences in 16S rRNA sequences

\begin{tabular}{|c|c|c|c|c|c|c|c|c|c|c|c|}
\hline \multirow[b]{2}{*}{$\operatorname{Taxon}(\mathrm{a})$} & \multicolumn{11}{|c|}{ Level of nucleotide differences or $\%$ sequence similarity ${ }^{a}$} \\
\hline & 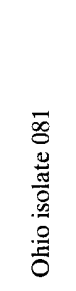 & 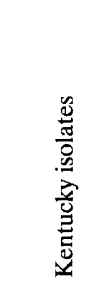 & 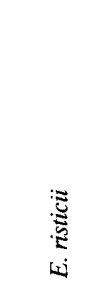 & 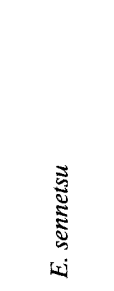 & 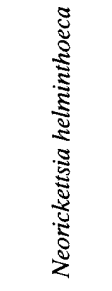 & 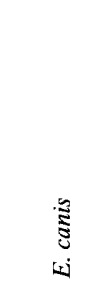 & 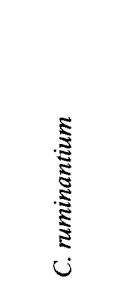 & 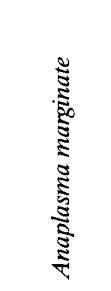 & 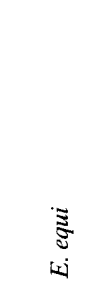 & 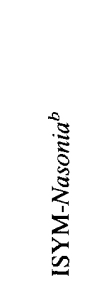 & 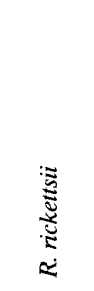 \\
\hline Ohio isolate 081 & & 0.0064 & 0.0071 & 0.0072 & 0.0465 & 0.1781 & 0.1777 & 0.1702 & 0.1688 & 0.1817 & 0.1905 \\
\hline Kentucky isolates & 99.37 & & 0.0035 & 0.0086 & 0.0454 & 0.1795 & 0.1791 & 0.1726 & 0.1683 & 0.1803 & 0.1924 \\
\hline E. risticii & 99.30 & 99.65 & & 0.0078 & 0.0462 & 0.1815 & 0.1792 & 0.1727 & 0.1684 & 0.1823 & 0.1938 \\
\hline E. sennetsu & 99.29 & 99.15 & 99.22 & & 0.0494 & 0.1776 & 0.1753 & 0.1719 & 0.1656 & 0.1801 & 0.1921 \\
\hline Neorickettsia helminthoeca & 95.52 & 95.62 & 95.65 & 95.26 & & 0.1597 & 0.1615 & 0.1719 & 0.1598 & 0.1771 & 0.1883 \\
\hline E. canis & 84.41 & 84.69 & 84.15 & 84.43 & 85.81 & & 0.0267 & 0.0825 & 0.0808 & 0.1440 & 0.1877 \\
\hline C. ruminantium & 84.43 & 84.32 & 84.32 & 84.60 & 85.67 & 97.39 & & 0.0805 & 0.0787 & 0.1422 & 0.1787 \\
\hline Anaplasma marginale & 85.02 & 84.83 & 84.83 & 84.88 & 84.89 & 92.29 & 92.48 & & 0.0350 & 0.1498 & 0.1818 \\
\hline E. equi & 85.12 & 85.15 & 85.15 & 85.36 & 85.82 & 92.44 & 92.63 & 96.63 & & 0.1382 & 0.1815 \\
\hline ISYM-Nasonia & 84.16 & 84.27 & 84.12 & 84.28 & 84.47 & 87.12 & 87.28 & 86.67 & 87.56 & & 0.1890 \\
\hline R. rickettsii & 83.37 & 83.22 & 83.12 & 83.25 & 83.53 & 83.62 & 84.29 & 84.07 & 84.08 & 83.57 & \\
\hline
\end{tabular}

${ }^{a}$ The values on the upper right are corrected levels of nucleotide differences for common pairwise homologous sites for up to 1,457 sites; the values on the lower left are maximum levels of sequence similarity determined from pairwise alignments.

${ }^{b}$ ISYM-Nasonia, intracellular endosymbionts of Nasonia sp.

following three groups of isolates were identified: (i) a set of five Ohio isolates, whose sequences were identical to the sequence of the $E$. risticii Illinois strain; (ii) isolate 081 , which had a unique sequence; and (iii) three Kentucky isolates, whose sequences were identical to each other, but different from the sequences of the members of the first two groups. The level of sequence divergence between isolate 081 and the Illinois strain was $0.7 \%$, the level of sequence divergence between the Kentucky isolates and the Illinois strain was $0.4 \%$, and the level of sequence divergence between the Kentucky isolates and isolate 081 was $0.6 \%$. These results were consistent with the results of previous immunoblot and IFA assays. In the previous antigenic analysis, isolate 081 was also significantly different from the

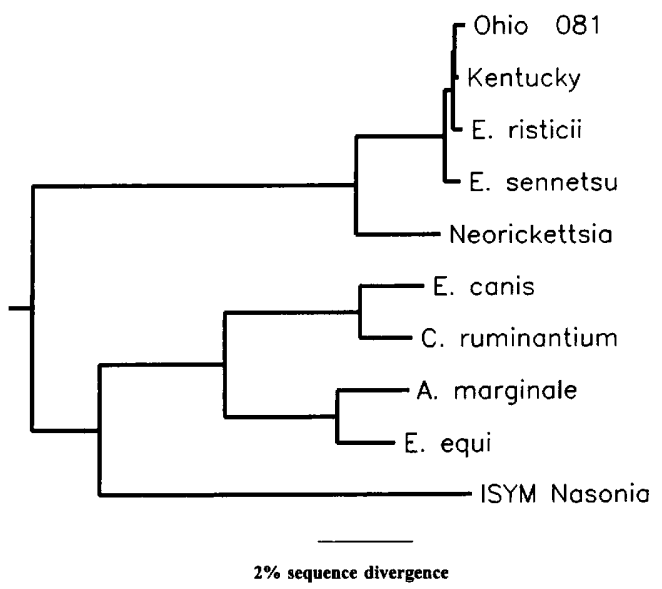

FIG. 2. Phylogenetic relationships among Ohio isolate 081, the Kentucky isolates, the E. risticii Illinois strain, various species belonging to the tribe Ehrlichieae, and the intracellular symbiont of Nasonia sp. (ISYM Nasonia). The tree was constructed by using the neighbor-joining method (NEIGHBOR in PHYLIP) from a matrix of corrected nucleotide divergence values. The evolutionary distance (percentage of nucleotide divergence) between a pair of species was calculated by adding the distances along the horizontal axis from the last common ancestor of the terminal taxa.
Illinois strain (3). The level of sequence divergence between isolate 081 and $E$. sennetsu was $0.7 \%$, which is identical to the level of sequence divergence between isolate 081 and the Illinois strain. On the basis of its distinct antigenic profiles and levels of $16 \mathrm{~S}$ rRNA divergence, isolate 081 is as distinct as $E$. sennetsu.

Do these levels of sequence divergence suggest that the isolates which we studied belong to three distinct Ehrlichia species? In related intracellular bacteria, the levels of $16 \mathrm{~S}$ rRNA gene divergence have often been found to be low. For instance, in an analysis of the 16S rRNA base sequences of members of the genus Ehrlichia, Anderson et al. (1) found that the levels of divergence between species ranged from 0.1 to $16 \%$. The levels of divergence between the $16 \mathrm{~S}$ rRNA sequences of several Rickettsia species have been found to be less than $0.4 \%$ (18). In another study, Czajka et al. (5) found only seven base differences in the 16S rRNA sequences of Listeria monocytogenes and Listeria innocua (level of divergence, $0.5 \%)$.

Differences between strains belonging to the same species are usually low. For instance, the 16S rRNA sequences of different strains of Rickettsia bellii were found to be identical (17). The levels of divergence between two E. sennetsu strains and between two $E$. canis strains were found to be only $0.1 \%$ (1). The sequences of different strains of $C$. ruminantium and of different strains of Anaplasma marginale reported in the GenBank database differ by only one nucleotide, while the sequences of different strains of Agrobacterium tumefaciens differ by zero to three nucleotides. The $16 \mathrm{~S}$ rRNA sequences of nine L. monocytogenes strains differ from each other by zero to five bases (5). Furthermore, the five nucleotide differences found in the most different $L$. monocytogenes strains are clustered in two close regions (nucleotides 194 to 196 and 214 to 215) (5). In contrast, however, the five bases which differentiate the Kentucky isolates from the Illinois strain of $E$. risticii are at five widely separated positions (nucleotides 97, 131, 956, 1221, and 1246). Therefore, it appears likely that the Kentucky isolates represent a species distinct from isolate 081 , although 
this remains to be demonstrated by other genotypic and phenotypic studies.

The genomic 16S rRNA gene groups based on the data presented above and previously were almost identical to the phenotypic (antigen) divisions of the nine isolates determined previously (3), suggesting that the $16 \mathrm{~S}$ rRNA and antigen genes might have coevolved, as would be expected if these strains represent genetically isolated forms bordering on species. E. sennetsu Miyayama strain was not found to be pathogenic for horses (14). In contrast to E. sennetsu, all nine isolates which we studied are considered virulent in horses since they were isolated from horses which had similar clinical signs of Potomac horse fever. Since the average substitution rate for $16 \mathrm{~S}$ rRNA in eubacteria is about $1 \%$ per $50 \times 10^{6}$ years $(8)$, the ehrlichial genetic divergence which we observed could have occurred millions of years ago, obviously before the recognition of Potomac horse fever. The clustering of the majority of the Ohio isolates together with the Illinois isolate and separate from the Kentucky isolates suggests that the 16S rRNA gene divergence observed is related to the geographic distribution of these organisms.

According to the $16 \mathrm{~S}$ rRNA signature positions of the different genetic groups, oligonucleotide probes or PCR primers can be designed to identify specific $E$. risticii groups by Southern blot analysis or PCR. By performing 16S rRNA gene analyses of additional isolates obtained from horses diagnosed with Potomac horse fever in the United States and other countries, we can increase our understanding of the relationships between genetic and antigenic divergence and geographic distribution of ehrlichiae which cause Potomac horse fever.

\section{ACKNOWLEDGMENT}

This study was supported in part by U.S. Department of Agriculture Section 1433 Animal Health and Disease Formula Funds.

\section{REFERENCES}

1. Anderson, B. E., J. E. Dawson, D. C. Jones, and K. H. Wilson. 1991. Ehrlichia chaffeensis, a new species associated with human ehrlichiosis. J. Clin. Microbiol. 29:2838-2842.

2. Cabot, E. L., and A. T. Bechenbach. 1989. Simultaneous editing of multiple nucleic acid and protein sequences with ESEE. Comput. Appl. Biosc. 5:233234.

3. Chaichanasiriwithaya, W., Y. Rikihisa, S. Yamamoto, S. M. Reed, T. B. Crawford, L. E. Perryman, and G. H. Palmer. 1994. Antigenic, morphologic, and molecular characterization of new Ehrlichia risticii isolates. J. Clin. Microbiol. 38:3026-3033.

4. Chen, S. M., J. S. Dumler, J. S. Bakken, and D. H. Walker. 1994. Identification of a granulocytotropic Ehrlichia species as the etiologic agent of human disease. J. Clin. Microbiol. 32:589-595.

5. Czajka, J., N. Bsat, M. Piani, W. Russ, K. Sultana, M. Wiedmann, R. Whiaker, and C. A. Batt. 1993. Differentiation of Listeria monocytogenes and Listeria innocua by $16 \mathrm{~S}$ rRNA genes and intraspecies discrimination of Listeria monocytogenes strains by random amplified polymorphic DNA polymorphism. Appl. Environ. Microbiol. 59:304-308.

6. Felsenstein, J. 1989. PHYLIP—phylogeny inference package (version 3.3). Cladistics 5:164-166.

7. Knowles, R. C., D. W. Anderson, W. D. Shipley, R. H. Whitlock, B. D. Perry, and J. D. Davidson. 1983. Acute equine diarrhea syndrome (AEDS): a preliminary report. Proc. Am. Assoc. Equine Pract. 29:353-357.

8. Ochman, H., and A. C. Wilson. 1987. Evolution in bacteria: evidence for a universal substitution rate in cellular genomes. J. Mol. Evol. 26:74-86.

9. Olsen, G. J., and C. R. Woese. 1993. Ribosomal RNA: a key to phylogeny. FASEB J. 7:113-123.

10. Palmer, J. E., R. H. Whitlock, and C. E. Benson. 1986. Equine ehrlichial colitis (Potomac horse fever): recognition of the disease in Pennsylvania, New Jersey, New York, Ohio, Idaho, and Connecticut. J. Am. Vet. Med. Assoc. 189:197-199.

11. Rikihisa, Y. 1991. The tribe Ehrlichieae and ehrlichial diseases. Clin. Microbiol. Rev. 4:286-308.

12. Rikihisa, Y. 1991. Cross-reacting antigens between Neorickettsia helminthoeca and Ehrlichia spp. shown by immunofluorescence and Western immunoblotting. J. Clin. Microbiol. 29:2024-2029.

13. Rikihisa, Y., S. A. Ewing, J. C. Fox, A. G. Siregar, F. H. Pasaribu, and M. B. Malole. 1992. Analysis of Ehrlichia canis and a canine granulocytic Ehrlichia infection. J. Clin. Microbiol. 30:143-148.

14. Rikihisa, Y., C. I. Pretzman, G. C. Johnson, S. M. Reed, S. Yamamoto, and F. Andrews. 1988. Clinical and immunological responses of ponies to Ehrlichia sennetsu and subsequent Ehrlichia risticii challenge. Infect. Immun. 56:2960-2966.

15. Ristic, M. 1990. Current strategies in research on ehrlichiosis, p. 136-153. In J. C. Williams and I. Kakoma (ed.), Ehrlichiosis: a vector-borne disease of animals and humans. Kluwer Academic Publishers, Boston.

16. Shankarappa, B., S. K. Dutta, and B. L. Mattingly-Napier. 1992. Antigenic and genomic relatedness among Ehrlichia risticii, Ehrlichia sennetsu, and Ehrlichia canis. Int. J. Syst. Bacteriol. 42:127-132.

17. Stothard, D. R., J. B. Clark, and P. A. Fuerst. 1994. Ancestral divergence of Rickettsia bellii from the spotted fever and typhus groups of Rickettsia and antiquity of the genus Rickettsia. Int. J. Syst. Bacteriol. 44:798-804

18. Stothard, D. R., and P. A. Fuerst. Evolutionary analysis of the spotted fever and typhus groups of Rickettsia using 16S rRNA gene sequences. Syst. Appl. Microbiol., in press

19. Vidor, E., G. Bissuel, Y. Moreau, J. L. Madec, and J. L. Cador. 1988 Serologie positive a Ehrlichia risticii chez une jument presentant un tableau d'ehrlichiose equine. Pratique Vet. Equine 20:5-10.

20. Weisburg, W. G., M. E. Dobson, J. E. Samuel, G. A. Dasch, L. Mallavia, O. Baca, L. Mandelco, J. E. Sechrest, E. Weiss, and C. R. Woese. 1989. Phylogenetic diversity of the rickettsiae. J. Bacteriol. 171:4202-4206.

21. Woese, C. R. 1987. Bacterial evolution. Microbiol. Rev. 51:221-271. 